
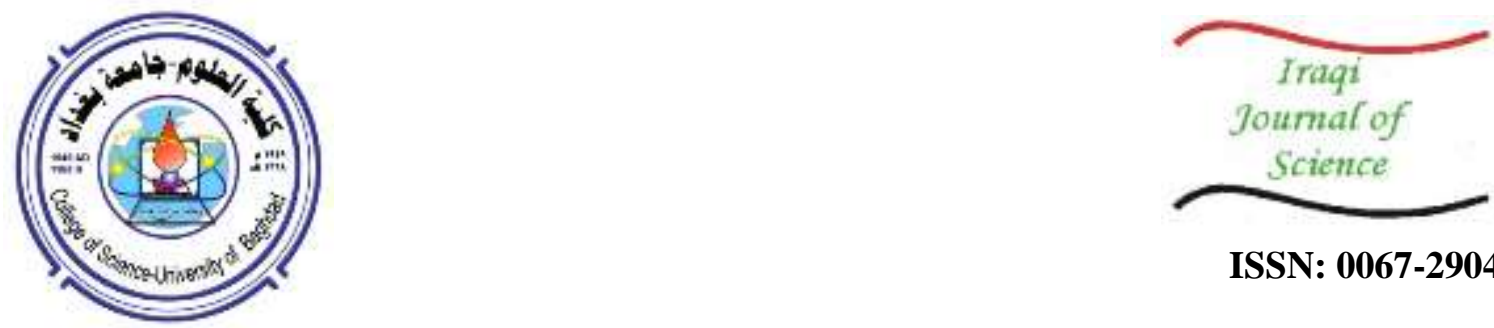

ISSN: 0067-2904

\title{
The convergence of Iteration Scheme to Fixed Points in Modular Spaces
}

\author{
Meena Fouad Abduljabbar*, Salwa Salman Abed \\ Department of mathematics, College of Education for Pure Science, Ibn Al-Haitham, University of Baghdad, \\ Baghdad, Iraq
}

\begin{abstract}
The aim of this paper is to study the convergence of an iteration scheme for multi-valued mappings which defined on a subset of a complete convex real modular. There are two main results, in the first result, we show that the convergence with respect to a multi-valued contraction mapping to a fixed point. And, in the second result, we deal with two different schemes for two multivalued mappings (one of them is a contraction and other has a fixed point) and then we show that the limit point of these two schemes is the same. Moreover, this limit will be the common fixed point the two mappings.
\end{abstract}

Key Words: Modular spaces, fixed points, multivalued mapping.

$$
\begin{aligned}
& \text { تقارب مخطط تكراري الى التقاط الثابتة في فضاء ات الوحدات } \\
& \text { مينة فؤاد عبد الجبار، سلوى سلمان عبد } \\
& \text { قسم الرياضيات، كلية التربية للعلوم الصرفة ابن الهيثث، جامعة بغداد، بغداد، العراق } \\
& \text { الخلاصة } \\
& \text { ان الهدف من هذا البحث هو دراسة التقارب لمخطط تكراري للتطبيقات متعددة القيم المعرفة على } \\
& \text { مجموعة جزئية من فضاء وحدات حقيقي كامل ومحدب. تم تقديم نتيجتين, في الاولى تم البرهنة على تقارب لتصن }
\end{aligned}
$$

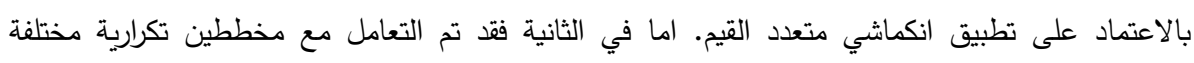

$$
\begin{aligned}
& \text { لتطبيقين متعددة القيم (واحد منها انكماشي والاخر يمتلك نقطة صامدة ) ثم البرهنة على ان المخططين } \\
& \text { تمتلكان نفس نقطة التقارب بالاضافة الى كونها نقطة صامدة مشتركة لكلا التطبيقين. }
\end{aligned}
$$

\section{1-Introduction and Preliminaries}

The notion of modular spaces was introduced by Nakano [1] in 1950 as a generalization of metric spaces and then redefined and modified by Musielak and Ortiz [2] in 1959. Many results about fixed points in these spaces were considered such as [3- 6]. Further and the most complete development of these theories are due to Orlicz, Mazur, Musielak, Luxemburg,Turpin [7] and their collaborators. In the present time the theory of modular and modular spaces is extensively applied, in particular, in the study of various Orlicz spaces [8], which in their turn have broad applications[9]. Recently, Abed [10] defined the best approximation and proved results about proximinal set, Chebysev set and existence invariant best approximation. see also [11] Now, recall the following

Definition 1.1[3] Let $M$ be a linear space over $F(=R$ or $\mathbb{W})$. A function $\gamma: M \rightarrow[0, \infty]$ is called modular if
(i) $\quad \gamma(v)=0$ if and only if $v=0$;
(ii) $\gamma(\alpha v)=\alpha(v)$ for $\alpha \in F$ with $|\alpha|=1$, for all $\alpha \in M$;
(iii) $\quad \gamma(\alpha v+\beta u) \leq \gamma(v)+\gamma(u)$ Iff $\alpha, \beta \geq 0$, for all $u, v \in M$.

*Email: mena.momo1961@gmail.com 
If (iii) replaced by

(iii)' $\gamma(\alpha v+\beta u) \leq \alpha \gamma(v)+\beta \gamma(u)$, for $\alpha, \beta \geq 0, \alpha+\beta=1$, for all $v, \mathrm{u} \in M$, Then $M_{\gamma}$ is called convex modular.

Definition 1.2 [3] A modular $\gamma$ defines a corresponding modular space, i.e.,the spac $M_{\gamma}$ given by

$$
M_{\gamma}=\{v \in M: \gamma(\alpha v) \rightarrow 0 \text { whenever } \alpha \rightarrow 0\} .
$$

Remark 1.1[4] By condition (iii) above, if $\mathrm{u}=0$ then $\gamma(\alpha v)=\gamma\left(\frac{\alpha}{\beta} \beta v\right) \leq \gamma(\beta v)$, for all $\alpha, \beta$ in $F$, $0<\alpha<\beta$. This shows that $\gamma$ is an increasing function.

\section{Example [12]}

The Orlicz modular is defined for every measurable real function $f$ by the formula(1) $\gamma(f)=$ $\int \varphi(|f(t)|) d m(t)$

Where $m$ denotes the Lebesgue measure in $R$ and $\varphi: R \rightarrow[0, \infty)$ is continuous in which $\varphi(u)=$ 0 if and only if $u=o$ and $\varphi(t) \rightarrow \infty$ as $n \rightarrow \infty$. The modular space induced by the Orlicz modular $\gamma_{\varphi}$ is called the Orlicz space $L^{\varphi}$

(2) The Musielak- Orlicz modular space . Let $\gamma(f)=\int \varphi(\omega, f(\omega)) d \mu(\omega)$,

Where $\mu$ is a $\sigma-$ finite measure on $\Omega$, and $\varphi: \Omega \times R \rightarrow[0, \infty)$ satisfy the following :

(i) $\varphi(\omega, u)$ is a continuous even function of $u$ which is nondecreasing for $u>0$ , Such that $\varphi(\omega, 0)=0, \varphi(\omega, u)>0$ for $u \neq 0$ and $\varphi(\omega, u) \rightarrow \infty$ as $n \rightarrow \infty$

(ii) $\varphi(\omega, u)$ is a measurable function of $\omega$ for each $u \in R$

Definition 1.3 [6] The $\gamma$-ball, $B_{r}(u)$ centered at $u \in M_{\gamma}$ with radius $r>0$ as $B_{r}(u)=\left\{\boldsymbol{v} \in M_{\gamma} ; \gamma(u-v)<r\right\}$.

The class of all $\gamma$-balls in a modular space $M_{\gamma}$ generates a topology which makes $M_{\gamma}$ Hausdorff topological linear space. Every $\gamma$-ball is convex set, therefore every modular space is locally convex Hausdorff topological vector space [7].

Definition 1.5 [6] Let $M_{\gamma}$ be a modular spase.

(a) A sequence $\left\{v_{n}\right\} \subset M_{\gamma}$ is said to be $\gamma$-convergent to $v \in M_{\gamma}$ and write $v_{n} \stackrel{\gamma}{\rightarrow} v$ if $\gamma\left(v_{n}-v\right) \rightarrow$ 0 as $n \rightarrow \infty$.

(b) A sequence $\left\{v_{n}\right\}$ is called $\gamma$-Cauchy whenever $\gamma\left(v_{n}-v_{m}\right) \rightarrow 0$ as $n, m \rightarrow \infty$.

(c) $M_{\gamma}$ is called $\gamma$-complete if any $\gamma$-Cauchy sequence in $M_{\gamma}$ is $\gamma$ - convergent.

(d) A subset $B \subset M_{\gamma}$ is called $\gamma$ - closed if for any sequence $\left\{v_{n}\right\} \subset B$ is $\gamma$-convergent to a point in $B$

(e) A $\gamma$-closed subset $B \subset M_{\gamma}$ is called $\gamma$-compact if any sequence $\left\{v_{n}\right\} \subset B$ has a $\gamma$-convergent subsequence.

(f) A subset $B \subset M_{\gamma}$ is said to be $\gamma$ - bounded if $\operatorname{daim}_{\gamma}(B)<\infty$, where

$$
\operatorname{daim}_{\gamma}(B)=\sup \{\gamma(v-u) ; v, u \in B\}
$$

is called the $\gamma$ - diameter of $B$.

(g) The distance between $v \in M_{\gamma}$ and $B \subset M_{\gamma}$ is

$\gamma(v-B)=\inf \{\gamma(v-u) ; u \in B\}$.

Definition 1.6 Let $M_{\gamma}$ be a modular space, and $A, B$ are two non - empty subsets in $2^{M_{\gamma}}$. Let $H_{\gamma}(A, B)$ denotes Hausdorff distance of $A$ and $B$ that is defined as the following

$H_{\gamma}(A, B)=\max \left\{\sup _{a \in A} \gamma(a-B), \sup _{b \in B} \gamma(b-A)\right\}$.

Lemma (1.1) Let $M_{\gamma}$ be a modular space and let $A_{n}$ and $B_{n}$ real sequences in $C B\left(M_{\gamma}\right)$ Then we can choose $a_{n}$ in $A_{n}, b_{n}$ in $B_{n}$ such that

$$
\gamma\left(a_{n}-b_{n}\right)=H_{\gamma}\left(A_{n}, B_{n}\right)+\varepsilon_{n}, \lim _{n \rightarrow \infty} \varepsilon_{n}=0 \quad \ldots(1.1)
$$

Definition (1.7) [13] or [14] Let $A$ be a non-empty set and $T: A \longrightarrow 2^{A}$ be a multi-valued mapping, the point $x \in A$ is said to be a fixed point of $T \Leftrightarrow x \in T(x)$. And $x$ is a fixed point of a single-valued mapping $T$ if and only if $x=T(x)$

Definition (1.8) [15] A mapping $T: A \rightarrow 2^{M_{\gamma}}$ such that

$$
H_{\gamma}(T u, T v) \leq K \gamma(u-v) \text { for all } u, v \in A \ldots .(1.2)
$$

is said to be multi - valued Lipschitz if there exists $k>0$ and multi - valued contraction (shortly, m.v.c) if $k<1$. 
The following lemmas are required in the next section.

Lemma (1.2): [15] Let $\left\{a_{n}\right\} \subset R^{+}$such that $a_{n+1} \leq\left(1-\lambda_{n}\right) a_{n}+\sigma_{n}$ where $\lambda_{n} \in(0,1)$, for all $n \in N, \quad \sum_{n=1}^{\infty} \lambda_{n}$ and $\sigma_{n}=o\left(\lambda_{n}\right)$. Then $\lim _{n \rightarrow \infty} a_{n}=0$.

Lemma (1.3): [16,17] Let $\left\{a_{n}\right\} \subset R^{+}$such that $a_{n+1} \leq\left(1-\lambda_{n}\right) a_{n}+\lambda_{n} \varepsilon$

where $\lambda_{n} \in(0,1)$, for all $n \in N, \quad \sum_{n=1}^{\infty} \lambda_{n}=\infty$ and $\varepsilon>0$ is fixed number. Then $0 \leq$ $\lim _{n \rightarrow \infty} \sup _{n} \leq \varepsilon$.

exists a $\delta(\varepsilon)>0$, such that

if $\gamma(v)=\gamma(u)=1$ and $(v-u) \geq \varepsilon$, then $\gamma\left(\frac{1}{2}(v+u)\right) \leq 1-\delta$.

\section{Main Results}

Let $A$ be a non-empty subset of $M_{\gamma}$, and $T: A \longrightarrow 2^{A} u_{0} \in A$. If the sequence $\left\{u_{n}\right\} \subset A$ is defined by

$$
u_{n+1} \in\left(1-a_{n}\right) u_{n}+a_{n} T v_{n}
$$

$v_{n} \in\left(1-\beta_{n}\right) u_{n}+\beta_{n} \mathrm{~T} u_{n}, \forall n \geq 0$

or

$\begin{array}{cr}u_{n+1}=\left(1-a_{n}\right) u_{n}+a_{n} \mu_{n}, & \mu_{n} \in T v_{n}, \forall n \geq 0 \\ v_{n}=\left(1-\beta_{n}\right) u_{n}+\beta_{n} \xi_{n}, \quad \xi_{n} \in T u_{n}, \forall n \geq 0 & (1.4)\end{array}$

$$
v_{n}=\left(1-\beta_{n}\right) u_{n}+\beta_{n} \xi_{n}, \quad \xi_{n} \in T u_{n}, \forall n \geq 0 \quad \ldots(1.4)
$$

Two convergence results for iteration (1.4) are established dealing with contraction. We start with the following needed lemma:-

Theorem (2.2) : Let $M_{\gamma}$ be a complete modular space and $\emptyset \neq A \subseteq M_{\gamma} A$ is a nonempty closed. If $T: A \rightarrow C B(A)$ is $m . v . c$ mapping, then $T$ has a fixed in $A$

Proof

Select $u_{0} \in A$ and $u_{1} \in T\left(u_{0}\right)$ By Lemma (1.1) there must exist $u_{2} \in T\left(u_{1}\right)$ such that

$$
\gamma\left(u_{1}-u_{2}\right) \leq H_{\gamma}\left(T\left(u_{0}\right), T\left(u_{1}\right)\right)+K
$$

similary, there exists $u_{3} \in T\left(u_{2}\right)$ such that

$$
\gamma\left(u_{2}-u_{3}\right) \leq H_{\gamma}\left(T\left(u_{1}\right), T\left(u_{2}\right)\right)+K^{2}
$$

By induction, there is the sequence $\left\{u_{n}\right\}$ in $A$ such that $\forall i, \in N, u_{i+1} \in T\left(u_{i}\right)$ and

Therefore ,

$$
\gamma\left(u_{i}-u_{i+1}\right) \leq k^{i} d\left(u_{0}, u_{1}\right)+i k^{i}
$$

$$
\sum_{i=0}^{\infty} \gamma\left(u_{i}-u_{i+1}\right) \leq \gamma\left(u_{0}-u_{1}\right)\left(\sum_{i=0}^{\infty} k^{i}\right)+\sum_{i=0}^{\infty} i k^{i}
$$

This prove that $\left\{u_{n}\right\}$ is a cauchy sequence. so, since $A$ is complete there exist $u \in A$ such that $\lim _{\mathrm{n} \rightarrow \infty} \mathrm{u}_{\mathrm{n}}=\mathrm{u}$. Also, the continuity of $T$ lied to $\lim _{n \rightarrow \infty} H_{\gamma}\left(T\left(u_{n}\right), T(u)\right)=0$

Since $u_{n} \in T\left(u_{n-1}\right)$, then

This implies that

$$
\lim _{n \rightarrow \infty} \operatorname{dis} t\left(u_{n}, T(u)\right)=\lim _{n \rightarrow \infty} \inf \left\{\gamma\left(u_{n}-v\right): v \in T(u)\right\}=0
$$

$\operatorname{dist}(u, T(u))=\inf \{\gamma(u-v): v \in T(u)\}=0$ And, the closeness of $T(u)$, it must be the case that $u \in T(u)$

Theorem (2.3) Let $M_{\gamma}$ be a complete convex real modular space, let $\emptyset \neq A \subseteq M_{\gamma}$ and $A$ be convex and closed subset of $M_{\gamma}$ and $\mathrm{T}: \mathrm{A} \longrightarrow \mathrm{CB}(\mathrm{A})$ be a m.v.c mapping. Let $\left\{\alpha_{n}\right\},\left\{\beta_{n}\right\} \subseteq$ $(0,1)$ satisfying:

(i) $0<\alpha_{n}, \beta_{n}<1$

(ii) $\sum_{n=1}^{\infty} \alpha_{n}=\infty$

then for $u_{0} \in A$, the sequence $\left\{u_{n}\right\}$ in (1.4) converges to a fixed point of $T$.

\section{Proof:}

The existence of the fixed point follows from the Theorem (2.2). Let $p \in A$ be a fixed point of $T$.

By conditions (1.4), (1.1) and (1.2), we get

$$
\begin{aligned}
& u_{n+1}-p=\left(1-\alpha_{n}\right) u_{n}+\alpha_{n} \mu_{n}-p \quad \text { where } \mu_{n} \in T v_{n} \\
& \gamma\left(u_{n+1}-p\right)=\gamma\left(\left(1-\alpha_{n}\right) u_{n}+\alpha_{n} \mu_{n}-\left(\left(1-\alpha_{n}\right) p+\alpha_{n} p\right)\right) \\
& =\gamma\left(\left(1-\alpha_{n}\right)\left(u_{n}-p\right)+\alpha_{n}\left(\mu_{n}-p\right)\right. \\
& \leq\left(1-\alpha_{n}\right) \gamma\left(u_{n}-p\right)+\alpha_{n} \gamma\left(\mu_{n}-p\right)
\end{aligned}
$$




$$
\begin{aligned}
& \leq\left(1-\alpha_{n}\right) \gamma\left(u_{n}-p\right)+\alpha_{n} H_{\gamma}\left(T v_{n}, T p\right)+\alpha_{n} \varepsilon_{n} \\
& \leq \quad\left(1-\alpha_{n}\right) \gamma\left(u_{n}-p\right)+\alpha_{n} k \gamma\left(v_{n}-p\right)+\alpha_{n} \varepsilon_{n}
\end{aligned}
$$

Again, from conditions (1.4), (1.1) and (1.2), we get

$$
\begin{aligned}
& \gamma\left(\quad u_{n+1}-p\right)=\left(1-\alpha_{n}\right) \gamma\left(u_{n}-p\right)+\alpha_{n} k \gamma\left(\left(1-\beta_{n}\right) u_{n}+\beta_{n} \varepsilon_{n}-\left(1-\beta_{n}\right) p-\beta_{n} p\right)+ \\
& \quad \alpha_{n} \varepsilon_{n} \quad \text { where } \varepsilon_{n} \in T u_{n} \\
& \quad \leq\left(1-\alpha_{n}\right) \gamma\left(u_{n}-p\right)+\alpha_{n} k\left(1-\beta_{n}\right) \gamma\left(u_{n}-p\right)+\alpha_{n} k \beta_{n} \gamma\left(\varepsilon_{n}-p\right)+\alpha_{n} \varepsilon_{n} \\
& \quad \leq\left(1-\alpha_{n}\right) \gamma\left(u_{n}-p\right)+\alpha_{n} k\left(1-\beta_{n}\right) \gamma\left(u_{n}-p\right)+\alpha_{n} k \beta_{n} H_{\gamma}\left(T \quad u_{n}, T p\right)+\alpha_{n} \varepsilon_{n}+ \\
& \alpha_{n} k \beta_{n} \varepsilon_{n} \\
& \leq\left(1-\alpha_{n}\right) \gamma\left(u_{n}-p\right)+\alpha_{n} k\left(1-\beta_{n}\right) \gamma\left(u_{n}-p\right)+\alpha_{n} k^{2} \beta_{n} \gamma\left(u_{n}-p\right)+\alpha_{n} \varepsilon_{n}+\alpha_{n} k \beta_{n} \varepsilon_{n} \\
& \quad \leq\left(\left(1-\alpha_{n}\right)+\alpha_{n} k\right) \gamma\left(u_{n}-p\right)+\alpha_{n} \varepsilon_{n}+\alpha_{n} k \beta_{n} \varepsilon_{n}
\end{aligned}
$$

Thus,

$\gamma\left(u_{n+1}-p\right)=\left(\left(1-\alpha_{n}(1-k)\right) p\left(u_{n}-p\right)+0\left(\alpha_{n}\right)\right.$

Let us denote

$a_{n}=\gamma\left(u_{n}-p\right)$

$\lambda_{n}=\alpha_{n}(1-k) \in(0,1) \quad \forall n \geq 0$

And using lemma ( 1.2 ), we obtain $\lim _{n \rightarrow \infty} a_{n}=0$. which implies that $\lim _{n \rightarrow \infty} \gamma\left(u_{n}-p\right)=$ 0 , thus $\lim _{n \rightarrow \infty} u_{n}=p$

Theorem (2.4): Let $M_{\gamma}$ be a complete convex real modular space, let $\emptyset \neq A \subseteq M_{\gamma}$ and $A$ be a convex and closed subset of $M_{\gamma}$. Let $\varepsilon>0$ be a fixed number and $T, S: A \longrightarrow C(A)$ be two multivalued mappings. If $\mathrm{S}$ is a m.v.c mapping and $\lim _{n \rightarrow \infty} w_{n}=p$, where $p$ is a fixed point of $T$, for any given $w_{0} \in A$, the sequence $\left\{w_{n}\right\}$ in (1.4) with $\left\{\alpha_{n}\right\},\left\{\beta_{n}\right\}$ satisfying

(i) $0<\alpha_{n}, \beta_{n}<1$

(ii) $\sum_{n=1}^{\infty} \alpha_{n}=\infty$

and if $H(T z, S z) \leq \epsilon$, for all $z \in A$, then $\mathrm{p}$ is a common fixed point of $T$ and $S$.

Proof:

The existence of the fixed point $q$ of $S$ follows from the Theorem (2.2). For the mapping $S$ from (1.4) be

$u_{n+1}=\left(1-\alpha_{n}\right) u_{n}+\alpha_{n} \theta_{n}, \quad$ where $\theta_{n} \in S v_{n}, \forall n \geq 0$

$v_{n}=\left(1-\beta_{n}\right) u_{n}+\beta_{n} \xi_{n}, \quad$ where $\xi_{n} \in S u_{n}, \forall n \geq 0$

So, from conditions (1.1) and (1.2), we get

$w_{n+1}-u_{n+1}=\left(1-\alpha_{n}\right)\left(w_{n}-u_{n}\right)+\alpha_{n}\left(\mu_{n}-\theta_{n}\right), \quad$ where $\theta_{n} \in S v_{n}$

$\gamma\left(w_{n+1}-u_{n+1}\right)=\gamma\left(\left(1-\alpha_{n}\right)\left(w_{n}-u_{n}\right)+\alpha_{n}\left(\mu_{n}-\theta_{n}\right)\right)$

$\leq\left(1-\alpha_{n}\right) \gamma\left(w_{n}-u_{n}\right)+\alpha_{n} \gamma\left(\mu_{n}-\theta_{n}\right)$

$=\left(1-\alpha_{n}\right) \gamma\left(w_{n}-u_{n}\right)+\alpha_{n} \gamma\left(\mu_{n}-\varpi_{n}+\varpi_{n}-\theta_{n}\right)$, where $\varpi_{n} \in S g_{n}$

$\leq\left(1-\alpha_{n}\right) \gamma\left(w_{n}-u_{n}\right)+\alpha_{n} \gamma\left(\mu_{n}-\varpi_{n}\right)+\alpha_{n} \gamma\left(\varpi_{n}-\theta_{n}\right)$

$\leq\left(1-\alpha_{n}\right) \gamma\left(w_{n}-u_{n}\right)+\alpha_{n} H_{\gamma}\left(T g_{n}, S g_{n}\right)+\alpha_{n} H_{\gamma}\left(S g_{n}, S v_{n}\right)+\alpha_{n} b_{n}+\alpha_{n} d_{n}$

$=\left(1-\alpha_{n}\right) \gamma\left(w_{n}-u_{n}\right)+\alpha_{n} \varepsilon+\alpha_{n} k \gamma\left(g_{n}-v_{n}\right)+\alpha_{n} b_{n}+\alpha_{n} d_{n}$

$\left.=\left(1-\alpha_{n}\right) \gamma\left(w_{n}-u_{n}\right)+\alpha_{n} \varepsilon+\alpha_{n} k \gamma\left(\left(1-\beta_{n}\right) w_{n}+\beta_{n} \xi_{n}-\left(1-\beta_{n}\right) u_{n}+\beta_{n} \zeta_{n}\right)\right) .+\alpha_{n} b_{n}+$ $\alpha_{n} d_{n}$

where $\xi_{n} \in T w_{n}, \zeta_{n} \in S u_{n}$

$=\left(1-\alpha_{n}\right) \quad \gamma\left(w_{n}-\mathrm{u}_{\mathrm{n}}\right)+\alpha_{n} \varepsilon+\alpha_{n} k \gamma\left(\left(1-\beta_{n}\right)\left(w_{n}-u_{n}\right)+\beta_{n}\left(\xi_{n}-\zeta_{n}\right)+\alpha_{n} b_{n}+\right.$ $\alpha_{n} d_{n}$

$\gamma\left(w_{n+1}-\mathrm{u}_{\mathrm{n}+1}\right) \leq\left(1-\alpha_{n}\right) \quad \gamma\left(w_{n}-\mathrm{u}_{\mathrm{n}}\right)+\alpha_{n} \varepsilon+\alpha_{n} k\left(1-\beta_{n}\right) \gamma\left(w_{n}-u_{n}\right)+$ $\alpha_{n} k \beta_{n} \gamma\left(\xi_{n}-\zeta_{n}\right)+\alpha_{n} b_{n}+\alpha_{n} d_{n}$

$=\left(1-\alpha_{n}\right) \gamma\left(w_{n}-\mathrm{u}_{\mathrm{n}}\right)+\alpha_{n} \varepsilon+\alpha_{n} k\left(1-\beta_{n}\right) \gamma\left(w_{n}-u_{n}\right)+\alpha_{n} k \beta_{n} \gamma\left(\xi_{n}-e_{n}+e_{n}-\right.$ $\left.\zeta_{n}\right)+\alpha_{n} b_{n}+\alpha_{n} d_{n}, \quad$ where $e_{n} \in S w_{n}$

$\leq\left(1-\alpha_{n}\right) \gamma\left(w_{n}-\mathrm{u}_{\mathrm{n}}\right)+\alpha_{n} \varepsilon+\alpha_{n} k\left(1-\beta_{n}\right) \gamma\left(w_{n}-u_{n}\right)+\alpha_{n} k \beta_{n} \gamma$ 


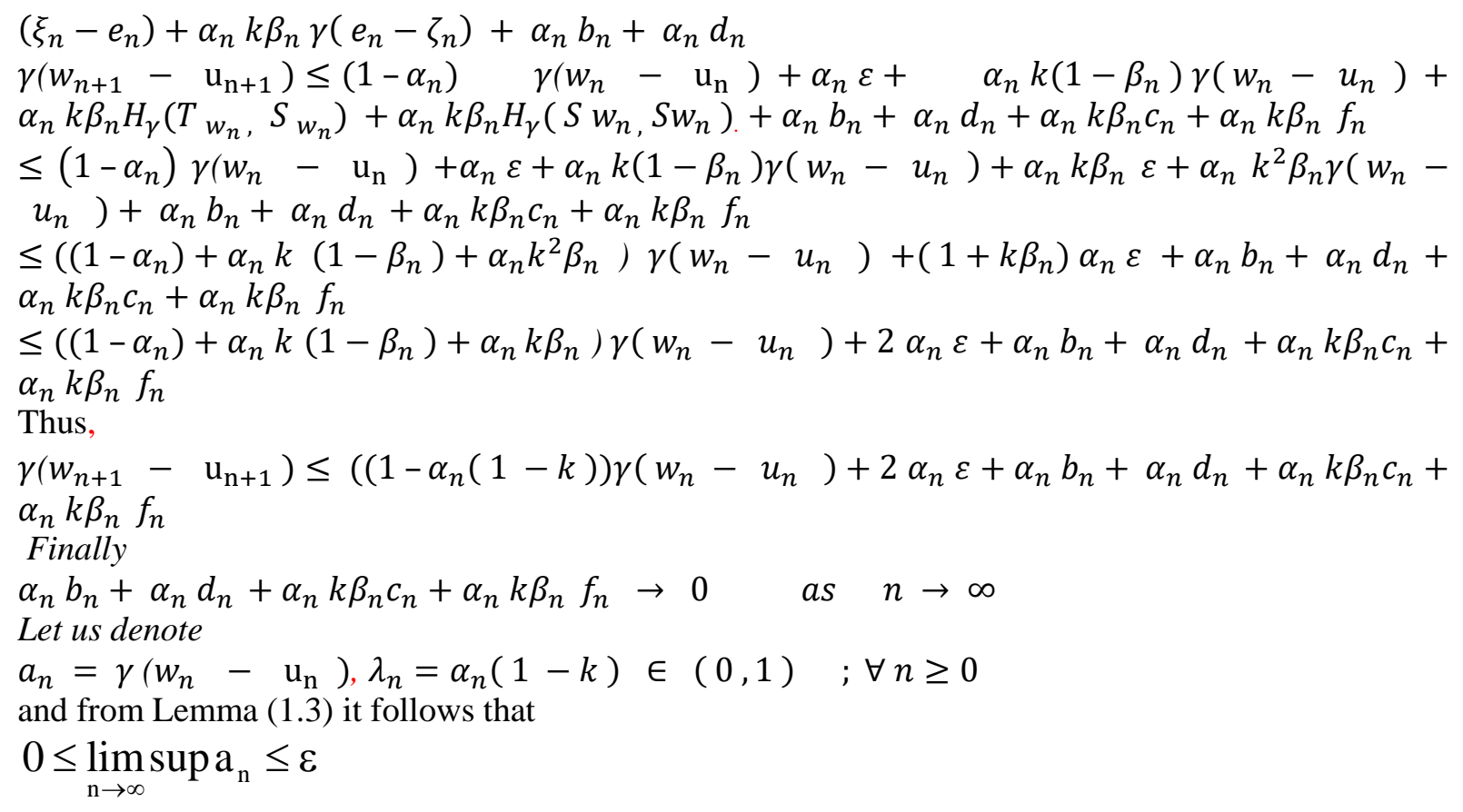

Since $\varepsilon$ is arbitrary $\lim _{n \rightarrow \infty} s u p a_{n}=0$ and so $\lim _{n \rightarrow \infty} a_{n}=0$, which implies that, and $\lim _{n \rightarrow \infty} \gamma\left(x_{n}-u_{n}\right)=0$, and $\lim _{n \rightarrow \infty} \gamma\left(x_{n}-u_{n}\right)=\gamma(p-q)$ so $\gamma(p-q)=0$, hence $p=q$.

Corollary (1):

Consider we have $M_{\gamma}, A$ and $T$ as in Theorem (2.2). For any $u_{0} \in A, u_{n+1}=\left(1-\alpha_{n}\right) u_{n}+$ $\alpha_{n} v_{n}, v_{n} \in T u_{n}, n \geq 0$

and $\left\{\alpha_{n}\right\} \subset R^{+}$satisfying:

(i) $0<\alpha_{n}<1$

(ii) $\sum_{n=1}^{\infty} \alpha_{n}=\infty$

the iteration sequence $\left\{u_{n}\right\}$ converges to a fixed point of $T$.

Proof: Follows from Theorem (2.3) with $\beta_{\mathrm{n}}=0 . \mathrm{n} \geq 0$

By the proof of Theorem (2.2), we have that the iteration sequence $u_{n+1}=T u_{n}$ converges to a fixed point of a $m . v . c$. mapping $T$.

\section{Corollary (2):}

Let $M_{\gamma}, A$ and $T$ as in Theorem (2.3). Let $\varepsilon>0$ be a fixed number. If $\mathrm{T}, \mathrm{S}: \mathrm{M} \longrightarrow \mathrm{C}(\mathrm{M})$ are two m.v.c. mappings, $\left\{u_{n}\right\}$ defined by condition (1.4) with $\left\{\alpha_{n}\right\},\left\{\beta_{n}\right\}$ satisfying

(i) $\quad 0<\alpha_{n}, \beta_{n}<1$

(ii) $\sum_{n=1}^{\infty} \alpha_{n}=\infty$

and if $H_{\gamma} \quad(T z, S z)$ for all $z \in M$, then $p$ is a common fixed point of $T$ and $S$.

\section{Proof:}

Since $T, S$ are m.v.c. mappings then $T, S$ have a fixed point $p, q$, and by Theorem (2.3) the iteration $\left\{u_{n}\right\}$ in condition (1.4) converges to $p$ and then by Theorem (2.4)

$\gamma(p-q)=0$

Hence

$p=q$

So, $p$ is a common fixed point of $T$ and $S$.

\section{References}

1. Nakano, H. 1950. "Modular semi-ordered linear spaces", in: Tokyo Math. Book Ser., Vol. 1, Maruzen Co., Tokyo.

2. Musielak, J. and Ortiz,W. 1959. " on modular spaces" , Studia Math, 18: 49-65.A

3. Abbas, M. S.H. Khan and Razani A. 2011. " Fixed point theorems of expansive type mappings in modular function spaces", Fixed Point Theory, 12(2): 235-240 
4. Kuaket, K. Kumam, P. 2011. " Fixed points of asymptotic point wise contractions in modular spaces" Appl. Math. Let. 24: 1795-1798

5. Kozlowski, WM. 2012. "Advancements in fixed point theory in modular function spaces", Arab J. Mat. 1: 477-494

6. Chen, R. Wang, X. 2013. "Fixed point of nonlinear contractions in modular spaces", J. of Ineq. And Appl. 2013.

7. Chistyakov, V. V. 2010. "Modular metric spaces", I: Basic concepts, Nonlinear Analysis, 72: 114

8. Kumam, P. 2004. Fixed point theorems for nonexpansive Mappings in modular spaces ,Archivum mathematicum ( Brno), Tomus, 40: 345 - 353

9. Rao, M.M. Ren, Z.D. 2004. " Applications of Orlicz Spaces", Pure Appl. Math., vol. 250, Marcel Dekker,

10. Abed, S.S. 2017. "On invariant best approximation in modular spaces", Glob. J. of Pure and Appl. Math. , 13(9): 5227-5233

11. Abed, S. S. Mohammed, N. J. 2018. "Best Approximation in Modular Spaces By Type of Nonexpansive Maps", Iraqi Journal of Science, 59(3B): 1450-1452

12. Khamsi , M . A. 1996. "Fixed point theory in modular function spacesm", Recent Advances on Metric Fixed point Theory, Universidad de sivilla, 8: 31 - 58

13. Zeidler, E. 1986. "Non linear function analysis and application"I . Fixed point theorems, Springer Verlage, New York

14. Rhoades, B. E. and Stefan, M .S. 2003. "The Equivalence between the convergences of Ishikawa and mann iteration for an Asymptotically Pseudo-Contractive map", J . Math . Appl . 283: $681-688$.

15. Smart, D. R. 1974. "Fixed point theorems", Cambridge University, New York

16. Park, J. Y and Jeong, J. U. 1996. "Ishikawa and Mann Iteration Methods for Strongly Accretive Operators", Comm . Korean Math, 13(4): 765 - 773.

17. Abed, S. S. and AbdulSada, K. E. 2018. " Approximatively Compactness and Best Approximation in Modular Spaces". Accepted in Conf. of Nahrain University 